\title{
Variability of optical Fe II complex in narrow-line Seyfert 1 galaxy NGC 4051
}

\author{
J. Wang ${ }^{1}$, J. Y. Wei ${ }^{2}$, and X. T. He \\ 1 National Astronomical Observatories, Chinese Academy of Sciences, Beijing 100012, China \\ e-mail: wjy@bao.ac.cn \\ 2 National Astronomical Observatories, Chinese Academy of Sciences, Beijing 100012, China \\ 3 Department of Astronomy, Beijing Normal University, Beijing 100875, China \\ e-mail: xthe@bun.edu.cn
}

Received 15 September 2004 / Accepted 24 January 2005

\begin{abstract}
The variability of optical Fe II blends in NGC 4051 is examined from spectra extracted from the AGN Watch program. In our analysis, the Fe II complex are subtracted and measured, with the following results. Firstly, the Fe II variations were detected in NGC 4051 during a 3-year period. The identified Fe II variations followed the variations in continuum closely. Secondly, the $E W$ of $\mathrm{Fe}$ II is reported to increase with rising continuum flux, which is consistent with previous claims that there is no convincing Baldwin Effect in optical Fe II. Thirdly, by comparing the variations of $\mathrm{H} \beta$ and Fe II, we find that $R_{\mathrm{Fe}}$ scales with continuum flux as $R_{\mathrm{Fe}} \propto(5.0 \pm 0.8) \log (L / M)$, which is significantly different from the theoretical expectations. Finally, in six selected Seyferts, four out of five Narrow-Line Seyfert 1 galaxies present positive correlation between $R_{\mathrm{Fe}}$ and the continuum flux. The negative correlations are identified in the remaining two objects that have relatively broad profiles of $\mathrm{H} \beta\left(F W H M>1500 \mathrm{~km} \mathrm{~s}^{-1}\right)$. We argue that the difference of electron density of broad line clouds and/or variability behavior of incident high-energy radiation can explain the dichotomy in variability behavior of $R_{\mathrm{Fe}}$.
\end{abstract}

Key words. galaxies: active - galaxies: individual: NGC 4051 - galaxies: quasars: emission lines

\section{Introduction}

Generally, the optical/UV spectra of Active Galactic Nuclei (AGNs) are prominently featured by broad and intense emission lines. The relative strength and radiative mechanism of broad emission lines can be interpreted well by the traditional photoionization models of many dense $\left(\sim 10^{9-10} \mathrm{~cm}^{-3}\right)$ clouds. Those clouds with approximate cosmic abundance are photoionized and heated by a covered central source (e.g. Davidson \& Netzer 1979; Kwan \& Krolik 1981; Kwan 1984; Stasinska 1984; Collin-Souffrin et al. 1988; Korista et al. 1997). Despite repeated successes of the standard photoionization models, important problems still remain.

Although collisional ionization is carefully introduced, the photoionization models are frustrated in the aspect that accounts for the strength of Fe II emission in both UV and optical bands (Netzer \& Wills 1983; Wills et al. 1985; Joly 1987; Collin-Souffrin et al. 1986, 1988; Dultzin-Hacyan 1987). If the total flux of Fe II is several times larger than Ly $\alpha$ (Wills et al. 1985), and if its energy is derived from photoionization, the models indicate that the X-ray flux must then be comparable to or greater than the Lyman continuum flux. This seems unlikely, however, given our present understanding. Early calculations of Fe II emission predicted that the flux ratio of total Fe II to $\mathrm{H} \beta$ was no more than 8 . However, the observed $\mathrm{Fe} \mathrm{II} / \mathrm{H} \beta$ is more typical by 12 and approaches about 30 in a few sources with "super-strong" Fe II emission (Lawrence et al. 1988; Graham et al. 1996; Lipari 1994; Moran et al. 1996; Xia et al. 1999; Zhou et al. 2002). In order to interpret the intense Fe II emission, a number of additional excitation mechanisms have been proposed. Sigut \& Pradhan (1998) suggested that the Ly $\alpha$ fluorescent excitation of Fe II could double Fe II strength in both UV and optical bands effectively. Joly (1991, and references therein) put forward the idea that collisional excitation is the most likely process in AGN, however, did not include virtual calculations.

The Fe II emission in AGN has consistently attracted a great deal of attention, both because of the problem described above and because of its importance in investigating the underlying physics that drives the Eigenvector 1 (E1). The E1 introduced by principal component analysis (PCA, Boroson \& Green 1992, hereafter BG92) contains a strong anti-correlation between $F W H M$ of $\mathrm{H} \beta$ and the flux ratio of the optical Fe II complex to the broad component of $\mathrm{H} \beta$. At the present time, it is believed that the E1 plays a vital role in research of AGN phenomena (e.g. Boroson 2002; Sulentic et al. 2000a,b; Marziani et al. 2001; Zamanov \& Marziani 2002; Marziani et al. 2003a). Both observational and theoretical studies indicate that the E1 is most likely driven by some elementary parameters of AGN, 
such as the central black hole mass, the Eddington ratio, and even the orientation of accretion axis (Jarvis \& McLure 2002). Investigation of the complex Fe II emission in AGN is, therefore, essential for further understanding E1 correlations and whole AGN phenomenon.

Actually, so far the variability behavior of the Fe II complex in Seyfert galaxies has been poorly understood. In Mark 110, Kollatschny et al. (2001) reported that the permitted optical Fe II complex remained constant within $10 \%$ error over 10 years, while the forbidden $[\mathrm{FeX}] \lambda 6375$ line was variable. Similarly, in the Seyfert 1 galaxy NGC 5548 no significant variations of the optical Fe II blends (less than 20\%) were detected (Dietrich et al. 1993). On the contrary, the opposite result was reported in a long term optical variability watch program on Seyfert 1 galaxy NGC 7603 over a period of nearly 20 years (Kollatschny et al. 2000). This object displayed remarkable variability in the Fe II blends, with amplitudes on same order as for the $\mathrm{H} \alpha$ and He I lines. Giannuzzo \& Stirpe (1996) found that, out of 12 Narrow-Line Seyfert 1 galaxies (NLS1s), at least 4 of them presented a significant variability of the Fe II complex with percentage variations larger than $30 \%$. In addition, considerable variations of the Fe II complex (larger than $50 \%$ ) were reported in the other two Seyfert 1 galaxies Akn 120 and Fairall 9 (Kollatschny et al. 1981; Kollatschny \& Fricke 1985). It is likely that further individual investigation, as well as subsequently statistical analyses, would be essential to comprehend the physics governing the Fe II emission in AGN.

NGC 4051, a well-known NLS1 natured by both narrow permitted emission lines $\left(F W H M \approx 1100 \mathrm{~km} \mathrm{~s}^{-1}\right)$ and a low ratio for $[\mathrm{O} \mathrm{III}] / \mathrm{H} \beta$ (Osterbrock \& Pogge 1985), was monitored for three years, from January 1996 to July 1998, as part of the AGN Watch campaign (Peterson et al. 2000). Peterson and his colleagues reported the strong variabilities of intensity in $\mathrm{H} \beta$ and He II $\lambda 4686$. Variations of the $\mathrm{H} \beta$ line were found to lag behind the variations in continuum by $6 \pm 2-3$ days. The time delay associated with the Doppler width of $\mathrm{H} \beta$ yields a viral mass estimation of $\sim 1.1 \times 10^{6} M_{\odot}$ for the central black hole. They also found that the Fe II blends varied little, if at all, in the period spanning three years according to the rms spectra.

In this paper, we investigate the variability of the optical Fe II complex in NGC 4051 by performing a new spectral analysis on the AGN Watch archival spectra. Besides investigation of this individual object, a comparison of the variability behavior of Fe II between diverse AGNs is performed to reveal the underlying physics governing Fe II emission.

The paper is organized as follows. In Sect. 2, we describe the spectral measurements in detail. The analysis and immediate results are given in Sect. 3. In Sect. 4, we propose two possible explanations, and finally discuss the underlying implications.

\section{Spectra measurements}

The archival spectra in one-dimensional FITS format were downloaded from the AGN Watch Web Site: http:// www-astronomy .mps. ohio-state.edu/ agnwatch/. This archive contains a total of 123 spectra divided into two data sets (A and B). Generally, for the wavelength coverage, Set B

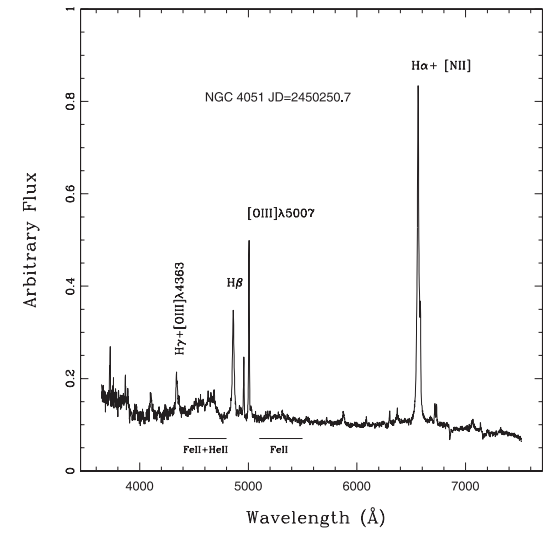

Fig. 1. Rest frame and Galactic extinction corrected spectrum of NGC 4051 taken on June 16, 1996 (JD = 2450250.7 ). The most prominent emission lines and optical Fe II complex are marked.

spectra extend shortward further than do spectra of Set A. In our analysis, we discard the first spectrum observed at Julian Date 2450095.0 because of the unavailable FITS file. The spectra n00229b and n00600b are obviated in measurements because of their poor spectral quality (i.e. bad $S / N$ for continuum, as well as for emission lines). Spectrum n00479a is excluded from spectral analysis, as well, because of its improper wavelength coverage only ranging between $4720 \AA$ and $5990 \AA$ A. Detailed information on the archival spectra can be consulted in Table 1 and Sect. 2.2 in Peterson et al. (2000). In Table 1, the file name of each of the remaining 120 spectra is given in Col. (1), and Col. (2) lists the corresponding Julian Date of observation.

\subsection{Pretreatments and Fe II measurements}

The remaining 120 spectra are pretreated in the following two steps: 1) the Galactic extinction is corrected by $E(B-$ $V)=0.013 \mathrm{mag}$ from NED, adopting an $R_{V}=3.1$ extinction curve; 2) each spectrum is transformed to rest frame, as well as K-corrected by the redshift derived by a Gaussian fitting for the narrow peak of the $\mathrm{H} \beta$ line. As an illustration, the rest frame spectrum of NGC 4051 taken on June 16, 1996 $(\mathrm{JD}=2450250.7)$ is shown in Fig. 1 . The spectrum covers the wavelength range from $3600 \AA$ to $7540 \AA$, and the strongest emission lines and prominent Fe II complex are labeled.

As shown in Fig. 1, there is a clear contribution from blends of $\mathrm{Fe}$ II line emission on both the red and blue sides of the $\mathrm{H} \beta$-[O III] complex. The blends contaminate strong emission lines and alter the fluxes of $\mathrm{H} \beta$ and $[\mathrm{O} \mathrm{III}] \lambda \lambda 4959,5007$. In order to determine contributions of the Fe II blends and to measure the other emission lines reliably, we subtract the Fe II multiplets from each observed spectrum by the experiential technique described by BG92. The subtraction depends on a template of the Fe II complex. In this paper, the adopted template is the same as that used in BG92, namely, the Fe II emission of IZW1, which is a well-known prototype of bright NLS1 with narrow permitted Fe II emission lines (Phillips 1978; Oke \& Lauer 1979). 
Table 1. Continuum and integrated line fluxes.

\begin{tabular}{|c|c|c|c|c|c|c|c|}
\hline $\begin{array}{c}\text { File name } \\
\text { (1) }\end{array}$ & $\begin{array}{c}\text { Julian Date }^{a} \\
\text { (2) }\end{array}$ & $\begin{array}{l}\text { Set } \\
(3)\end{array}$ & $\begin{array}{c}F_{\lambda}(5100 \AA)^{b} \\
(4)\end{array}$ & $\begin{array}{c}F\left(\mathrm{H} \beta_{\mathrm{P}}\right)^{c} \\
(5)\end{array}$ & $\begin{array}{c}F(\mathrm{Fe} \mathrm{II})^{c} \\
(6)\end{array}$ & $\begin{array}{c}F(\mathrm{H} \beta)^{d, e} \\
(7)\end{array}$ & $\begin{array}{c}F(\mathrm{He} \text { II } \lambda 4686)^{d, f} \\
(8)\end{array}$ \\
\hline $\mathrm{n} 00098 \mathrm{~b}$ & 98.0 & B & $13.88 \pm 0.56$ & $4.54 \pm 0.18$ & $6.94_{-1.39}^{+1.39}$ & $6.34 \pm 0.23$ & $5.13 \pm 0.19$ \\
\hline $\mathrm{n} 00102 \mathrm{~b}$ & 102.0 & B & $14.42 \pm 0.58$ & $4.91 \pm 0.20$ & $8.96_{-1.79}^{+2.39}$ & $6.81 \pm 0.21$ & $4.74 \pm 0.15$ \\
\hline $\mathrm{n} 00105 \mathrm{~b}$ & 105.1 & B & $13.46 \pm 0.54$ & $4.54 \pm 0.18$ & $7.82_{-1.20}^{+2.41}$ & $6.18 \pm 0.25$ & $3.02 \pm 0.16$ \\
\hline $\mathrm{n} 00108 \mathrm{~b}$ & $108.0 \ldots \ldots$ & B & $13.75 \pm 0.55$ & $4.39 \pm 0.17$ & $8.09_{-152}^{+2.02}$ & $6.06 \pm 0.30$ & $4.20 \pm 0.21$ \\
\hline $\mathrm{n} 00112 \mathrm{~b}$ & $112.0 \ldots \ldots$ & B & $13.75 \pm 0.55$ & $4.42 \pm 0.18$ & $7.45_{-1.60}^{+2.13}$ & $5.91 \pm 0.23$ & $4.92 \pm 0.18$ \\
\hline n00118a & $118.8 \ldots \ldots$ & A & $14.01 \pm 0.28$ & $4.20 \pm 0.08$ & $4.44_{-2.66}^{+1.33}$ & $5.23 \pm 0.20$ & $4.01 \pm 0.32$ \\
\hline $\mathrm{n} 00122 \mathrm{a}$ & $122.8 \ldots \ldots$ & A & $14.12 \pm 0.28$ & $4.67 \pm 0.09$ & $6.34_{-1.45}^{+2.93}$ & $5.64 \pm 0.20$ & $2.00 \pm 0.08$ \\
\hline n00123b & $124.0 \ldots \ldots$ & B & $13.74 \pm 0.55$ & $4.72 \pm 0.19$ & $8.01_{-1.50}^{+3.400}$ & $6.61 \pm 0.27$ & $4.00 \pm 0.19$ \\
\hline $\mathrm{n} 00127 \mathrm{a}$ & $127.8 \ldots \ldots$ & A & $13.51 \pm 0.27$ & $4.66 \pm 0.09$ & $6.41_{-1.97}^{+2.96}$ & $5.97 \pm 0.14$ & $2.42 \pm 0.09$ \\
\hline $\mathrm{n} 00129 \mathrm{~b}$ & 129.0 & B & $14.37 \pm 0.57$ & $5.09 \pm 0.20$ & $8.70_{-1.74}^{+2.32}$ & $7.07 \pm 0.25$ & $4.93 \pm 0.18$ \\
\hline $\mathrm{n} 00132 \mathrm{~b}$ & $132.0 \ldots \ldots$ & B & $14.91 \pm 0.60$ & $4.42 \pm 0.18$ & $9.14_{-2.74}^{+2.74}$ & $5.47 \pm 0.12$ & $3.52 \pm 0.20$ \\
\hline $\mathrm{n} 00134 \mathrm{~b}$ & 134.9 & B & $13.39 \pm 0.54$ & $4.63 \pm 0.19$ & $8.50_{-2.04}^{+2.04}$ & $6.61 \pm 1.22$ & $7.90 \pm 0.83$ \\
\hline n00136a & $136.8 \ldots \ldots$ & A & $14.13 \pm 0.28$ & $4.53 \pm 0.09$ & $7.63_{-1.09}^{+2.72}$ & $5.57 \pm 0.24$ & $3.07 \pm 0.25$ \\
\hline n00149a & $149.8 \ldots \ldots$ & A & $14.70 \pm 0.29$ & $4.91 \pm 0.10$ & $8.44_{-1.59}^{+3.17}$ & $6.23 \pm 0.23$ & $3.38 \pm 0.14$ \\
\hline n00164a & $164.8 \ldots \ldots$ & A & $14.29 \pm 0.29$ & $4.71 \pm 0.09$ & $7.75_{-2.45}^{+2.91}$ & $5.94 \pm 0.24$ & $3.24 \pm 0.13$ \\
\hline $\mathrm{n} 00167 \mathrm{~b}$ & $167.7 \ldots \ldots$ & B & $14.66 \pm 0.59$ & $4.92 \pm 0.20$ & $7.82_{-1.74}^{+3.48}$ & $6.14 \pm 0.22$ & $3.18 \pm 0.09$ \\
\hline n00168a & $168.8 \ldots \ldots$ & A & $14.58 \pm 0.29$ & $4.75 \pm 0.09$ & $7.15_{-1.53}^{+4.08}$ & $5.91 \pm 0.21$ & $2.76 \pm 0.09$ \\
\hline $\mathrm{n} 00170 \mathrm{~b}$ & $170.8 \ldots \ldots$ & B & $14.17 \pm 0.57$ & $5.06 \pm 0.20$ & $8.30_{-1.11}^{+3.32}$ & $6.44 \pm 0.35$ & $4.04 \pm 0.21$ \\
\hline $\mathrm{n} 00183 \mathrm{~b}$ & 183.6 & B & $14.66 \pm 0.59$ & $4.84 \pm 0.19$ & $8.35_{-1.67}^{+4.45}$ & $6.47 \pm 0.37$ & $4.05 \pm 0.16$ \\
\hline $\mathrm{n} 00185 \mathrm{~b}$ & $185.6 \ldots \ldots$ & B & $14.51 \pm 0.58$ & $5.29 \pm 0.21$ & $8.05_{-1.42}^{+3.79}$ & $6.91 \pm 0.26$ & $3.62 \pm 0.13$ \\
\hline n00185a & $185.7 \ldots \ldots$ & A & $14.06 \pm 0.28$ & $5.12 \pm 0.10$ & $8.24_{-1.55}^{+4.12}$ & $6.37 \pm 0.27$ & $1.73 \pm 0.09$ \\
\hline n00188b & $188.6 \ldots \ldots$ & B & $14.61 \pm 0.58$ & $5.17 \pm 0.21$ & $9.28_{-1.36}^{+2.78}$ & $7.23 \pm 0.30$ & $5.33 \pm 0.14$ \\
\hline n00191b & $191.6 \ldots \ldots$ & B & $14.63 \pm 0.58$ & $5.35 \pm 0.21$ & $8.63_{-1.73}^{+2.30}$ & $6.94 \pm 0.28$ & $4.37 \pm 0.15$ \\
\hline n00193b & $193.6 \ldots \ldots$ & B & $15.02 \pm 0.60$ & $5.75 \pm 0.23$ & $8.62_{-1.73}^{+2.30}$ & $7.36 \pm 0.29$ & $6.35 \pm 0.16$ \\
\hline n00198a & $198.7 \ldots \ldots$ & A & $14.43 \pm 0.29$ & $5.63 \pm 0.11$ & $8.56_{-2.14}^{+4.28}$ & $7.65 \pm 0.15$ & $3.70 \pm 0.12$ \\
\hline n00198b & $198.8 \ldots \ldots$ & B & $13.54 \pm 0.54$ & $5.55 \pm 0.22$ & $9.01_{-1.80}^{+2.70}$ & $6.64 \pm 0.64$ & $6.44 \pm 0.40$ \\
\hline $\mathrm{n} 00199 \mathrm{~b}$ & $199.6 \ldots \ldots$ & B & $14.02 \pm 0.56$ & $5.54 \pm 0.22$ & $8.26_{-1.13}^{+1.50}$ & $6.45 \pm 0.80$ & $6.61 \pm 0.55$ \\
\hline n00206a & $206.7 \ldots \ldots$ & A & $14.08 \pm 0.28$ & $5.63 \pm 0.11$ & $7.67_{-2.05}^{+3.07}$ & $7.37 \pm 0.22$ & $3.95 \pm 0.10$ \\
\hline $\mathrm{n} 00211 \mathrm{~b}$ & $211.6 \ldots \ldots$ & B & $13.54 \pm 0.54$ & $5.48 \pm 0.22$ & $7.97_{-1.14}^{+1.90}$ & $7.18 \pm 0.33$ & $4.62 \pm 0.21$ \\
\hline $\mathrm{n} 00212 \mathrm{a}$ & $212.8 \ldots \ldots$ & A & $13.15 \pm 0.26$ & $5.36 \pm 0.11$ & $7.48_{-1.87}^{+2.80}$ & $6.80 \pm 0.20$ & $2.84 \pm 0.11$ \\
\hline n00213b & $213.6 \ldots \ldots$ & B & $13.16 \pm 0.53$ & $5.28 \pm 0.21$ & $7.78_{-1.46}^{+1.95}$ & $6.91 \pm 0.48$ & $4.17 \pm 0.19$ \\
\hline $\mathrm{n} 00218 \mathrm{~b}$ & $218.6 \ldots \ldots$ & B & $12.95 \pm 0.52$ & $5.09 \pm 0.20$ & $9.27_{-2.42}^{+3.22}$ & $6.74 \pm 0.48$ & $4.20 \pm 0.26$ \\
\hline $\mathrm{n} 00220 \mathrm{a}$ & $220.8 \ldots \ldots$ & A & $12.27 \pm 0.25$ & $4.59 \pm 0.09$ & $6.43_{-2.14}^{+2.57}$ & $5.98 \pm 0.21$ & $2.90 \pm 0.17$ \\
\hline n00221b & $221.7 \ldots \ldots$ & B & $13.34 \pm 0.53$ & $4.28 \pm 0.17$ & $6.96_{-2.78}^{+2.78}$ & $6.11 \pm 0.17$ & $3.53 \pm 0.14$ \\
\hline $\mathrm{n} 00225 \mathrm{~b}$ & $225.6 \ldots \ldots$ & B & $12.26 \pm 0.49$ & $4.37 \pm 0.17$ & $7.59_{-1.79}^{+1.79}$ & $5.81 \pm 0.33$ & $3.04 \pm 0.18$ \\
\hline $\mathrm{n} 00227 \mathrm{a}$ & $227.8 \ldots \ldots$ & A & $13.14 \pm 0.26$ & $4.14 \pm 0.08$ & $5.73_{-3.53}^{+3.53}$ & $5.36 \pm 0.19$ & $3.54 \pm 0.11$ \\
\hline $\mathrm{n} 00233 \mathrm{a}$ & $233.7 \ldots \ldots$ & A & $12.84 \pm 0.26$ & $4.51 \pm 0.09$ & $6.41_{-1.97}^{+1.97}$ & $5.81 \pm 0.22$ & $2.43 \pm 0.11$ \\
\hline $\mathrm{n} 00240 \mathrm{~b}$ & $240.6 \ldots \ldots$ & B & $13.18 \pm 0.53$ & $4.88 \pm 0.19$ & $8.85_{-1.31}^{+2.62}$ & $6.34 \pm 0.61$ & $3.73 \pm 0.25$ \\
\hline n00241a & $241.7 \ldots \ldots$ & A & $13.29 \pm 0.27$ & $4.78 \pm 0.10$ & $7.46_{-1.87}^{+2.80}$ & $6.50 \pm 0.20$ & $3.74 \pm 0.74$ \\
\hline $\mathrm{n} 00248 \mathrm{a}$ & $248.7 \ldots \ldots$ & A & $13.93 \pm 0.28$ & $5.64 \pm 0.11$ & $7.64_{-2.04}^{+3.06}$ & $7.50 \pm 0.16$ & $3.95 \pm 0.09$ \\
\hline $\mathrm{n} 00250 \mathrm{~b}$ & $250.7 \ldots \ldots$ & B & $12.99 \pm 0.52$ & $5.68 \pm 0.23$ & $7.54_{-2.51}^{+1.67}$ & $7.52 \pm 1.12$ & $4.34 \pm 0.85$ \\
\hline $\mathrm{n} 00253 \mathrm{~b}$ & $253.6 \ldots \ldots$ & B & $11.67 \pm 0.47$ & $5.22 \pm 0.21$ & $7.31_{-0.86}^{+1.72}$ & $6.62 \pm 0.38$ & $3.29 \pm 0.20$ \\
\hline n00253a & $253.7 \ldots \ldots$ & A & $11.67 \pm 0.47$ & $5.22 \pm 0.21$ & $6.94_{-1.30}^{+2.60}$ & $6.50 \pm 0.26$ & $2.64 \pm 0.16$ \\
\hline $\mathrm{n} 00257 \mathrm{~b}$ & $257.7 \ldots \ldots$ & B & $12.09 \pm 0.48$ & $4.47 \pm 0.18$ & $6.45_{-2.15}^{+2.58}$ & $5.82 \pm 0.33$ & $2.81 \pm 0.16$ \\
\hline $\mathrm{n} 00259 \mathrm{~b}$ & 259.6 & B & $11.92 \pm 0.48$ & $4.57 \pm 0.18$ & $8.29_{-1.44}^{+1.44}$ & $6.38 \pm 0.34$ & $4.66 \pm 0.33$ \\
\hline $\mathrm{n} 00262 \mathrm{a}$ & $262.7 \ldots \ldots$ & A & $12.41 \pm 0.25$ & $4.17 \pm 0.08$ & $6.29_{-1.45}^{+2.90}$ & $5.35 \pm 0.17$ & $2.49 \pm 0.10$ \\
\hline n00281b & $281.6 \ldots \ldots$ & B & $13.64 \pm 0.55$ & $5.25 \pm 0.21$ & $10.76_{-1.62}^{+4.31}$ & $7.53 \pm 0.31$ & $4.98 \pm 0.22$ \\
\hline n00284b & $284.7 \ldots \ldots$ & B & $14.68 \pm 0.59$ & $5.89 \pm 0.24$ & $9.17_{-1.62}^{+3.24}$ & $7.78 \pm 0.26$ & $5.52 \pm 0.16$ \\
\hline n00288b & $288.6 \ldots \ldots$ & B & $13.25 \pm 0.53$ & $5.45 \pm 0.22$ & $9.97_{-2.30}^{+3.07}$ & $7.52 \pm 0.26$ & $4.17 \pm 0.19$ \\
\hline $\mathrm{n} 00429 \mathrm{~b}$ & $429.0 \ldots \ldots$ & B & $12.98 \pm 0.52$ & $5.48 \pm 0.23$ & $8.17_{-1.36}^{+2.72}$ & $7.65 \pm 0.38$ & $4.50 \pm 0.20$ \\
\hline $\mathrm{n} 00435 \mathrm{~b}$ & $435.0 \ldots \ldots$ & B & $13.53 \pm 0.54$ & $5.46 \pm 0.22$ & $9.44_{-2.46}^{+2.46}$ & $7.78 \pm 0.42$ & $5.36 \pm 0.32$ \\
\hline n00451b & $451.0 \ldots \ldots$ & $\mathrm{B}$ & $12.84 \pm 0.51$ & $5.51 \pm 0.22$ & $10.83_{-1.97}^{+1.97}$ & $7.66 \pm 0.38$ & $4.60 \pm 0.18$ \\
\hline $\mathrm{n} 00458 \mathrm{~b}$ & $458.0 \ldots \ldots$ & B & $13.25 \pm 0.53$ & $5.38 \pm 0.22$ & $9.99_{-1.36}^{+2.72}$ & $7.07 \pm 0.41$ & $4.23 \pm 0.20$ \\
\hline $\mathrm{n} 00465 \mathrm{~b}$ & $465.0 \ldots \ldots$ & B & $12.30 \pm 0.49$ & $4.74 \pm 0.19$ & $9.62_{-3.20}^{+3.66}$ & $7.04 \pm 0.27$ & $4.20 \pm 0.46$ \\
\hline n00480b & $480.0 \ldots \ldots$ & B & $12.68 \pm 0.51$ & $5.10 \pm 0.20$ & $9.17_{-2.04}^{+4.08}$ & $5.90 \pm 0.87$ & $3.80 \pm 1.00$ \\
\hline n00483b & $483.1 \ldots \ldots$ & B & $11.95 \pm 0.48$ & $4.69 \pm 0.19$ & $6.88_{-2.95}^{+2.95}$ & $5.92 \pm 0.76$ & $2.44 \pm 0.17$ \\
\hline n00485b & $485.9 \ldots \ldots$ & $\mathrm{B}$ & $12.41 \pm 0.50$ & $4.70 \pm 0.19$ & $6.31_{-3.15}^{+3.15}$ & $5.68 \pm 0.28$ & $3.30 \pm 0.12$ \\
\hline n00488b & $489.0 \ldots \ldots$ & B & $14.03 \pm 0.56$ & $4.92 \pm 0.20$ & $7.04_{-2.34}^{+1.56}$ & $6.03 \pm 0.48$ & $3.88 \pm 0.35$ \\
\hline n00493a & $493.1 \ldots \ldots$ & A & $12.67 \pm 0.25$ & $4.99 \pm 0.10$ & $6.61_{-1.42}^{+1.89}$ & $6.68 \pm 0.22$ & $4.59 \pm 0.17$ \\
\hline n00494b & $494.0 \ldots \ldots$ & B & $13.53 \pm 0.54$ & $4.83 \pm 0.19$ & $10.03_{-2.01}^{+4.01}$ & $6.67 \pm 0.23$ & $4.48 \pm 0.13$ \\
\hline n00506a & $506.8 \ldots \ldots$ & A & $11.46 \pm 0.23$ & $4.69 \pm 0.09$ & $5.44_{-1.17}^{+1.56}$ & $6.55 \pm 0.27$ & $2.60 \pm 0.21$ \\
\hline
\end{tabular}


Table 1. continued.

\begin{tabular}{|c|c|c|c|c|c|c|c|}
\hline $\begin{array}{c}\text { File name } \\
\text { (1) }\end{array}$ & $\begin{array}{c}\text { Julian Date }^{a} \\
\text { (2) }\end{array}$ & $\begin{array}{l}\text { Set } \\
(3)\end{array}$ & $\begin{array}{c}F_{\lambda}(5100 \AA)^{b} \\
(4)\end{array}$ & $\begin{array}{c}F\left(\mathrm{H} \beta_{\mathrm{P}}\right)^{c} \\
(5)\end{array}$ & $\begin{array}{c}F(\mathrm{Fe} \mathrm{II})^{c} \\
(6)\end{array}$ & $\begin{array}{c}F(\mathrm{H} \beta)^{d, e} \\
(7)\end{array}$ & $\begin{array}{c}F(\mathrm{He} \text { II } \lambda 4686)^{d, f} \\
(8)\end{array}$ \\
\hline n00509b & 510.0 . & $\mathrm{B}$ & $12.30 \pm 0.49$ & $4.37 \pm 0.17$ & $5.55_{-1.39}^{+1.85}$ & $5.09 \pm 0.53$ & $2.30 \pm 0.12$ \\
\hline n00519b & $519.8 \ldots \ldots$ & B & $12.51 \pm 0.50$ & $4.74 \pm 0.19$ & $6.58_{-1.56}^{+3.03}$ & $6.10 \pm 0.24$ & $3.80 \pm 0.17$ \\
\hline $\mathrm{n} 00520 \mathrm{a}$ & 520.8 . & A & $12.50 \pm 0.25$ & $5.12 \pm 0.10$ & $5.33_{-1.60}^{+1.60}$ & $6.65 \pm 0.16$ & $3.32 \pm 0.09$ \\
\hline n00521b & $521.8 \ldots \ldots$ & B & $12.65 \pm 0.51$ & $4.68 \pm 0.19$ & $6.39_{-1.24}^{+3.76}$ & $6.25 \pm 0.47$ & $3.70 \pm 0.24$ \\
\hline $\mathrm{n} 00527 \mathrm{a}$ & 527.8 . & A & $10.92 \pm 0.22$ & $4.57 \pm 0.09$ & $4.82_{-1.45}^{+1.93}$ & $5.75 \pm 0.16$ & $2.90 \pm 0.11$ \\
\hline n00547b & 547.6 & B & $11.80 \pm 0.47$ & $4.52 \pm 0.18$ & $5.66_{-1.06}^{+1.41}$ & $5.49 \pm 0.48$ & $2.29 \pm 0.17$ \\
\hline $\mathrm{n} 00550 \mathrm{~b}$ & $550.8 \ldots \ldots$ & B & $11.74 \pm 0.47$ & $4.47 \pm 0.18$ & $5.93_{-1.98}^{+1.98}$ & $5.65 \pm 0.38$ & $2.34 \pm 0.15$ \\
\hline n00551b & $551.8 \ldots \ldots$ & B & $11.59 \pm 0.46$ & $4.42 \pm 0.18$ & $5.29_{-1.92}^{+2.88}$ & $5.53 \pm 0.27$ & $2.32 \pm 0.13$ \\
\hline $\mathrm{n} 00567 \mathrm{~b}$ & 567.6 & B & $10.88 \pm 0.44$ & $4.48 \pm 0.18$ & $6.08_{-1.30}^{+3.47}$ & $5.77 \pm 0.30$ & $3.25 \pm 0.16$ \\
\hline $\mathrm{n} 00569 \mathrm{~b}$ & 569.6 . & B & $11.76 \pm 0.47$ & $4.31 \pm 0.17$ & $4.59_{-1.84}^{+1.84}$ & $5.30 \pm 0.31$ & $2.56 \pm 0.16$ \\
\hline n00578b & 578.6 & B & $12.02 \pm 0.48$ & $4.32 \pm 0.17$ & $5.16_{-1.82}^{+1.82}$ & $5.96 \pm 0.35$ & $2.70 \pm 0.27$ \\
\hline $\mathrm{n} 00580 \mathrm{~b}$ & 580.7 . & B & $11.97 \pm 0.48$ & $4.91 \pm 0.20$ & $4.41_{-1.65}^{+1.10}$ & $6.20 \pm 0.48$ & $3.13 \pm 0.29$ \\
\hline $\mathrm{n} 00582 \mathrm{~b}$ & $582.6 \ldots$ & B & $12.10 \pm 0.48$ & $4.49 \pm 0.18$ & $5.07_{-1.27}^{+1.69}$ & $5.69 \pm 0.43$ & $2.80 \pm 0.21$ \\
\hline n00597b & 597.7. & B & $12.57 \pm 0.50$ & $4.24 \pm 0.17$ & $4.27_{-2.57}^{+3.42}$ & $4.76 \pm 0.09$ & $1.60 \pm 0.10$ \\
\hline n00602b & $602.6 \ldots \ldots$ & B & $11.62 \pm 0.47$ & $4.61 \pm 0.18$ & $4.35_{-2.18}^{+2.61}$ & $6.07 \pm 0.34$ & $2.79 \pm 0.21$ \\
\hline n00604b & 604.6 . & B & $12.00 \pm 0.48$ & $4.50 \pm 0.18$ & $5.10_{-1.70}^{+1.70}$ & $5.22 \pm 0.14$ & $2.02 \pm 0.22$ \\
\hline n00608b & 608.6 . & B & $11.96 \pm 0.48$ & $4.16 \pm 0.17$ & $3.98_{-1.19}^{+1.59}$ & $4.81 \pm 0.12$ & $2.04 \pm 0.22$ \\
\hline n00610b & 610.6 & B & $10.99 \pm 0.44$ & $4.24 \pm 0.17$ & $5.99_{-1.38}^{+1.84}$ & $5.10 \pm 0.11$ & $1.99 \pm 0.20$ \\
\hline n00627b & $627.6 \ldots \ldots$ & B & $11.26 \pm 0.45$ & $4.01 \pm 0.16$ & $5.66_{-1.70}^{+3.40}$ & $4.77 \pm 0.38$ & $1.97 \pm 0.27$ \\
\hline n00630b & $630.7 \ldots \ldots$ & B & $11.14 \pm 0.45$ & $3.88 \pm 0.16$ & $4.35_{-2.61}^{+1.74}$ & $5.08 \pm 0.31$ & $2.60 \pm 0.19$ \\
\hline n00635b & $635.6 \ldots \ldots$ & B & $11.87 \pm 0.47$ & $4.24 \pm 0.17$ & $5.43_{-1.81}^{+3.62}$ & $5.48 \pm 0.66$ & $2.77 \pm 0.33$ \\
\hline n00641b & $641.6 \ldots \ldots$ & B & $12.33 \pm 0.49$ & $4.71 \pm 0.19$ & $6.36_{-2.73}^{+1.82}$ & $6.22 \pm 0.32$ & $3.30 \pm 0.16$ \\
\hline n00643b & $643.6 \ldots \ldots$ & B & $10.75 \pm 0.43$ & $4.46 \pm 0.18$ & $4.95_{-2.12}^{+2.83}$ & $5.66 \pm 0.18$ & $2.66 \pm 0.13$ \\
\hline n00775a & $775.0 \ldots \ldots$ & A & $13.29 \pm 0.27$ & $5.04 \pm 0.10$ & $6.29_{-2.41}^{+1.93}$ & $6.82 \pm 0.19$ & $3.11 \pm 0.11$ \\
\hline $\mathrm{n} 00777 \mathrm{~b}$ & 777.0 . & B & $12.69 \pm 0.51$ & $5.18 \pm 0.21$ & $6.53_{-2.34}^{+1.87}$ & $6.68 \pm 0.36$ & $3.11 \pm 0.14$ \\
\hline $\mathrm{n} 00782 \mathrm{~b}$ & $782.0 \ldots \ldots$ & B & $12.86 \pm 0.51$ & $5.41 \pm 0.22$ & $7.21_{-1.55}^{+2.06}$ & $6.78 \pm 0.25$ & $2.96 \pm 0.13$ \\
\hline n00787b & $787.0 \ldots \ldots$ & B & $13.49 \pm 0.54$ & $5.27 \pm 0.21$ & $7.39_{-1.59}^{+3.17}$ & $6.83 \pm 0.22$ & $3.83 \pm 0.12$ \\
\hline n00810b & 810.1 . & B & $14.06 \pm 0.56$ & $3.95 \pm 0.16$ & $7.11_{-1.19}^{+3.16}$ & $5.12 \pm 0.34$ & $2.22 \pm 0.19$ \\
\hline n00834b & $834.0 \ldots \ldots$ & B & $12.00 \pm 0.48$ & $4.85 \pm 0.19$ & $8.14_{-1.92}^{+4.79}$ & $5.65 \pm 0.20$ & $1.62 \pm 0.32$ \\
\hline n00838b & $838.1 \ldots \ldots$ & B & $11.44 \pm 0.46$ & $4.59 \pm 0.18$ & $5.93_{-3.20}^{+2.74}$ & $5.78 \pm 0.27$ & $2.23 \pm 0.13$ \\
\hline n00839a & $839.0 \ldots \ldots$ & A & $11.89 \pm 0.24$ & $4.89 \pm 0.10$ & $5.60_{-1.29}^{+1.29}$ & $6.31 \pm 0.25$ & $2.85 \pm 0.15$ \\
\hline n00840b & 840.1 . & B & $12.50 \pm 0.50$ & $4.91 \pm 0.20$ & $6.77_{-1.56}^{+2.08}$ & $5.85 \pm 0.27$ & $2.01 \pm 0.10$ \\
\hline n00843b & $843.0 \ldots \ldots$ & B & $12.45 \pm 0.50$ & $4.87 \pm 0.19$ & $5.74_{-2.78}^{+1.91}$ & $5.55 \pm 0.26$ & $1.62 \pm 0.14$ \\
\hline $\mathrm{n} 00847 \mathrm{~b}$ & 847.0 . & B & $12.33 \pm 0.49$ & $4.82 \pm 0.19$ & $7.12_{-1.90}^{+2.85}$ & $6.10 \pm 0.34$ & $2.14 \pm 0.14$ \\
\hline n00867b & $867.0 \ldots \ldots$ & B & $12.25 \pm 0.49$ & $4.20 \pm 0.17$ & $6.53_{-1.09}^{+2.18}$ & $4.95 \pm 0.59$ & $1.31 \pm 0.13$ \\
\hline n00869b & 869.0 . & B & $12.55 \pm 0.50$ & $3.85 \pm 0.15$ & $4.95_{-1.98}^{+1.98}$ & $4.79 \pm 0.27$ & $1.71 \pm 0.13$ \\
\hline $\mathrm{n} 00872 \mathrm{~b}$ & $873.0 \ldots \ldots$ & B & $12.15 \pm 0.49$ & $4.09 \pm 0.16$ & $4.74_{-2.64}^{+2.11}$ & $4.88 \pm 0.27$ & $1.93 \pm 0.10$ \\
\hline n00876a & 876.0 . & $\mathrm{A}$ & $12.58 \pm 0.50$ & $4.31 \pm 0.17$ & $\begin{array}{l}5.27_{-2.39}^{+2.39} \\
\end{array}$ & $5.78 \pm 0.20$ & $3.31 \pm 0.10$ \\
\hline n00877b & $877.0 \ldots \ldots$ & B & $12.44 \pm 0.50$ & $4.37 \pm 0.17$ & $6.11_{-1.67}^{+2.22}$ & $5.54 \pm 0.21$ & $2.13 \pm 0.11$ \\
\hline n00886a & $885.8 \ldots \ldots$ & A & $11.54 \pm 0.23$ & $4.18 \pm 0.08$ & $4.88_{-1.95}^{+1.95}$ & $5.43 \pm 0.20$ & $2.71 \pm 0.12$ \\
\hline n00892b & $892.8 \ldots \ldots$ & B & $12.14 \pm 0.49$ & $4.54 \pm 0.18$ & $5.83_{-1.94}^{+1.94}$ & $5.42 \pm 0.23$ & $1.85 \pm 0.11$ \\
\hline n00906b & $906.9 \ldots \ldots$ & B & $11.44 \pm 0.46$ & $4.53 \pm 0.18$ & $5.64_{-1.88}^{+2.82}$ & $5.73 \pm 0.27$ & $2.22 \pm 0.14$ \\
\hline n00921b & $921.9 \ldots \ldots$ & B & $11.61 \pm 0.46$ & $4.53 \pm 0.18$ & $6.35_{-2.93}^{+2.93}$ & $5.57 \pm 0.28$ & $1.91 \pm 0.12$ \\
\hline n00927a & $926.8 \ldots \ldots$ & A & $11.13 \pm 0.22$ & $4.43 \pm 0.09$ & $4.62_{-1.85}^{+1.85}$ & $5.81 \pm 0.17$ & $2.57 \pm 0.14$ \\
\hline n00930b & $930.8 \ldots \ldots$ & B & $11.43 \pm 0.46$ & $4.01 \pm 0.16$ & $4.89_{-1.87}^{+2.67}$ & $5.00 \pm 0.34$ & $1.74 \pm 0.15$ \\
\hline n00935b & $935.9 \ldots \ldots$ & B & $11.92 \pm 0.48$ & $4.51 \pm 0.18$ & $5.35_{-1.89}^{+1.89}$ & $5.68 \pm 0.40$ & $2.85 \pm 0.20$ \\
\hline n00936b & $936.6 \ldots \ldots$ & B & $11.23 \pm 0.45$ & $4.39 \pm 0.18$ & $5.30_{-2.66}^{+1.77}$ & $5.30 \pm 0.27$ & $1.84 \pm 0.11$ \\
\hline n00949b & $949.6 \ldots \ldots$ & B & $12.03 \pm 0.48$ & $5.04 \pm 0.20$ & $5.20_{-2.24}^{+1.49}$ & $6.06 \pm 0.19$ & $2.15 \pm 0.09$ \\
\hline n00961b & $961.6 \ldots \ldots$ & B & $11.32 \pm 0.45$ & $4.90 \pm 0.20$ & $6.29_{-1.46}^{+1.94}$ & $5.94 \pm 0.31$ & $2.32 \pm 0.11$ \\
\hline n00966b & $966.7 \ldots \ldots$ & B & $11.60 \pm 0.46$ & $4.39 \pm 0.18$ & $4.47_{-2.69}^{+2.24}$ & $5.23 \pm 0.24$ & $2.09 \pm 0.12$ \\
\hline n00981a & $980.7 \ldots \ldots$ & A & $11.72 \pm 0.21$ & $4.73 \pm 0.08$ & $5.88_{-1.47}^{+2.94}$ & $6.34 \pm 0.06$ & $2.79 \pm 0.04$ \\
\hline n00983b & $983.7 \ldots \ldots$ & B & $11.41 \pm 0.46$ & $4.46 \pm 0.18$ & $5.88_{-1.83}^{+2.20}$ & $5.37 \pm 0.32$ & $2.00 \pm 0.14$ \\
\hline n00988b & $988.6 \ldots \ldots$ & B & $11.56 \pm 0.46$ & $4.30 \pm 0.17$ & $5.26_{-2.39}^{+2.39}$ & $5.05 \pm 0.27$ & $1.72 \pm 0.10$ \\
\hline n00991b & $991.7 \ldots \ldots$ & B & $11.35 \pm 0.45$ & $4.15 \pm 0.17$ & $6.69_{-1.25}^{+2.09}$ & $5.07 \pm 0.24$ & $1.92 \pm 0.12$ \\
\hline n00994b & $994.6 \ldots \ldots$ & $\mathrm{B}$ & $11.37 \pm 0.46$ & $4.11 \pm 0.16$ & $6.48_{-1.50}^{+2.99}$ & $5.02 \pm 0.26$ & $1.49 \pm 0.13$ \\
\hline n01009b & $1009.6 \ldots$ & B & $10.98 \pm 0.44$ & $3.76 \pm 0.15$ & $4.50_{-2.25}^{+1.80}$ & $4.50 \pm 0.30$ & $1.55 \pm 0.13$ \\
\hline n01012b & $1012.6 \ldots$ & B & $12.18 \pm 0.49$ & $3.70 \pm 0.15$ & $5.71_{-1.55}^{+2.59}$ & $4.57 \pm 0.21$ & $1.79 \pm 0.12$ \\
\hline n01019b & $1019.6 \ldots$ & B & $12.75 \pm 0.51$ & $4.20 \pm 0.17$ & $5.89_{-2.72}^{+3.63}$ & $5.27 \pm 0.33$ & $2.80 \pm 0.15$ \\
\hline $\mathrm{n} 01022 \mathrm{~b}$ & $1022.6 \ldots$ & B & $12.09 \pm 0.48$ & $4.57 \pm 0.18$ & $7.26_{-3.63}^{+3.63}$ & $5.52 \pm 0.40$ & $1.58 \pm 0.16$ \\
\hline
\end{tabular}

${ }^{a}$ The Julian Date is scaled to zero point at JD $=2450000$

${ }^{b}$ In units of $10^{-15} \mathrm{erg} \mathrm{s}^{-1} \mathrm{~cm}^{-2} \AA^{-1}$. The values are obtained by Peterson et al. (2000).

${ }^{c}$ In units of $10^{-13} \mathrm{erg} \mathrm{s}^{-1} \mathrm{~cm}^{-2}$. The fluxes are obtained from Peterson et al. (2000).

${ }^{d}$ In units of $10^{-13} \mathrm{erg} \mathrm{s}^{-1} \mathrm{~cm}^{-2}$.

${ }^{e}$ Each flux includes the contributions of all three components.

${ }^{f}$ Each flux contains the contributions of a broad base and a narrow peak. 


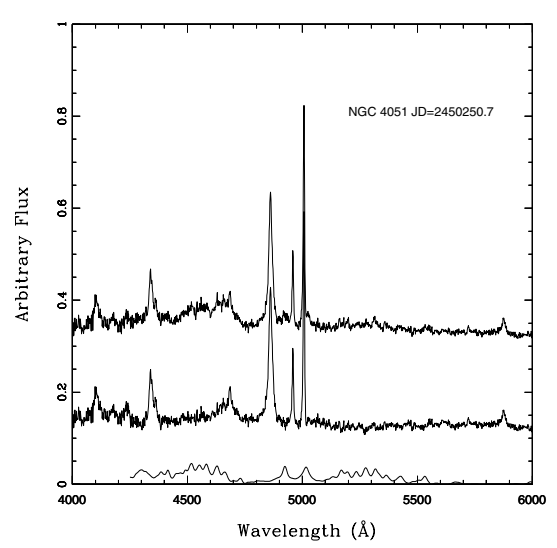

Fig. 2. The scheme of the Fe II complex subtraction of spectrum taken at JD $=2450250.7$. The top and middle curves are Fe II emission blended and subtracted spectra, respectively. The observed spectrum is shifted upward by an arbitrary amount. The bottom spectrum is the best adopted template of the Fe II complex.

The detailed procedure for making the template of the Fe II emission can be found in BG92, but, briefly, the template is a two-dimensional function of $F W H M$ and intensity of the Fe II blends. The template can be broadened to the FWHM of the broad component of $\mathrm{H} \beta$ by convolving with a Gaussian profile and scaled to match the Fe II strength. In NGC 4051, the $F W H M$ of the template is taken to be $1200 \mathrm{~km} \mathrm{~s}^{-1}$ which approximates the value of $F W H M$ of $\mathrm{H} \beta\left(V_{F W H M}=1110 \pm\right.$ $190 \mathrm{~km} \mathrm{~s}^{-1}$ ) derived by Peterson et al. (2000). For each spectrum, the scaling factor is estimated at rest wavelength $4570 \AA$; then the convolved and scaled templates are subtracted from the observed spectra. A successful Fe II subtraction requires a smooth continuum at blueward of $\mathrm{H} \beta$ and between $5100 \AA$ and $5500 \AA$. The Fe II subtraction is sketched in Fig. 2 for the case observed at JD $=2450250.7$. In the figure, the bottom curve is the best estimated Fe II multiplets; and the Fe II subtracted spectrum is shown in the middle; while the observed spectrum is plotted at the top. Note that the observed spectrum is offset upwards arbitrarily for visibility. The errorbar of Fe II strength, however, is very hard to obtained accurately because the subtraction is done by eye. Therefore, the uncertainties of Fe II intensities, which generally should be round about 30-50 percent, are superseded by the upper and lower limits. These limits are carefully obtained by iterative experimentations with a series of values for the flux of the template. Outside of the limits, the Fe II subtracted continuum is absolutely unacceptable.

A new Fe II template was recently published by Véron-Cetty et al. (2004). We perform the Fe II subtraction described above for 7 typical spectra in the terms of this new template. The inferred Fe II intensities are compared to the intensities provided by BG92's template. The two Fe II flux measurements are highly correlated and consistent with a nearly linear relation. This relation indicates that both templates can achieve the same result when we focus attention on the variability behavior of the optical Fe II complex. In addition to this relationship, the Fe II flux obtained by the template of Véron is systematically lower than the flux obtained by employing the BG92 template. For instance, in the spectrum observed at JD $=2450250.7$, the calibrated Fe II flux (see Sect. 3) is $5.25 \times 10^{-13} \mathrm{erg} \mathrm{s}^{-1} \mathrm{~cm}^{-2}$ for the Véron's template, but $7.54 \times 10^{-13} \mathrm{erg} \mathrm{s}^{-1} \mathrm{~cm}^{-2}$ for the BG92 template. This discrepancy is quite rational because the two templates differ in their narrow permitted and forbidden Fe II lines. These lines being of not negligible fluxes are excluded from the Véron's template.

\subsection{Line profile modelling}

The Fe II multiplets contamination-removed spectra are characterized not only by the prominent $\mathrm{H} \beta$ and [O III] $] \lambda 4959,5007$ lines, but also by their broad and strong He II $\lambda 4686$ emission. The next step in processing the spectra is to remove the continuum from each spectrum. Generally, the continuum is carefully modelled by a power law based upon two selected wavelength regions $4400 \AA-4450 \AA$ and $5100 \AA-5500 \AA$ in most situations. Both regions are free of any strong emission lines. The IRAF-SPECFIT task, a multi-component profile modelling procedure described by Kriss (1994), was utilized to model the isolated emission lines. Véron-Cetty et al. (2001) claimed that it is better to model $\mathrm{H} \beta$ emission line by a Lorentzian profile than by a Gaussian profile in NLS1 galaxies. The choice of profile to represent the observed emission line, however, may have no physical significance (e.g. Evans 1988; Xu et al. 2003), especially when we focus on an integrated line flux.

The following Gaussian components and specific relationships are involved when modelling each of the spectra. For the first step, the profile of each of the forbidden [O III] $\lambda \lambda 4959,5007$ lines is synthesized from a narrow core, as well as from a broad and blueshifted base (e.g. Heckman et al. 1981; Véron-Cetty et al. 2001; Zamanov et al. 2002; Christopoulou et al. 1997). The atomic physical relationships $F_{5007} / F_{4959} \doteq 3$ (Storey \& Zeippen 2001) and $\lambda_{4959} / \lambda_{5007}=0.9904$ are employed to decrease the number of free parameters in the modelling of both narrow and broad components. As a second step the $\mathrm{H} \beta$ line profile is synthesized from both a narrow Gaussian component and a broader one. Although this representation can fit the $\mathrm{H} \beta$ line core well and agree with a generally accepted unified model for AGN, it could not fit the far blue wing of $\mathrm{H} \beta$ adequately. Therefore, an additional blueshifted Gaussian component with $F W H M \sim 6000 \mathrm{~km} \mathrm{~s}^{-1}$ should be acquired to fit the far blue wing of $\mathrm{H} \beta$ (e.g. Sulentic et al. 2000c; Marziani et al. 2003b; Korista \& Goad 2004). In summary, a set of the following three Gaussian components are adopted to model the $\mathrm{H} \beta$ profile: a narrow core, a classical broad component with $F W H M \sim 1200 \mathrm{~km} \mathrm{~s}^{-1}$, and a very broad and blueshifted base. The $F W H M$ of narrow $\mathrm{H} \beta$ is taken to be equal to that of the [O III] core, because both components are emitted from the same region, i.e. from the narrow line region (NLR). The He II $\lambda 4684$ line profile is easily fitted by a set of two Gaussian profiles because of its substantial reflection in profile. As an illustration, the profile modelling of the spectrum observed at JD $=2450250.7$ is shown schematically in Fig. 3 . 


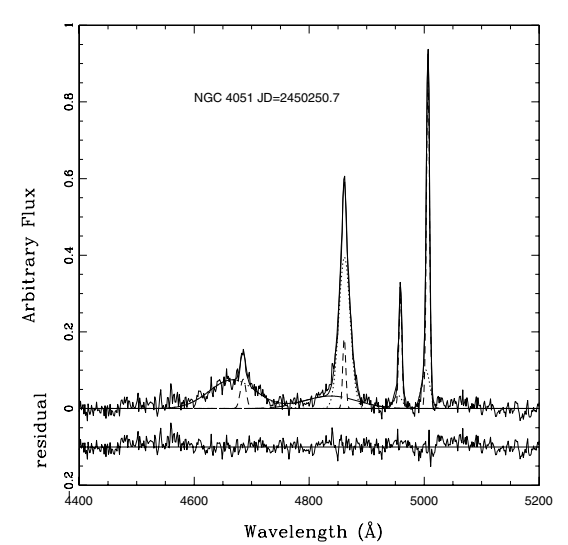

Fig. 3. An illustration of line-profile modelling of a continuumremoved spectrum taken on June 16,1996 (JD $=2450250.7)$. The observed profile is shown by the thin solid line, and the modelled profile, by the thick solid line. The narrow and broad components of each emission feature are represented by long and short dashed lines, respectively. The residuals of profile modelling are displayed in the lower panel.

The observed profile is represented by a thin line, and the modelled profile by a solid line. Each Gaussian component is shown by either a long- or short-dashed line. The residuals between the observed and modelled profile are presented in the bottom panel underneath the spectrum.

\section{Results}

The modelled flux of each component is calibrated by a constant total flux of $[\mathrm{O} \mathrm{III}] \lambda 5007$, where $F([\mathrm{O} \mathrm{III}] \lambda 5007)=$ $(3.91 \pm 0.12) \times 10^{-13} \mathrm{erg} \mathrm{s}^{-1} \mathrm{~cm}^{-2}$ (Peterson et al. 2000). The small systematical flux difference between the two sets (set A and B) are corrected by Formulas 5 and 6 in Peterson et al. (2000).

The final results of profile modelling, along with the continuum and total $\mathrm{H} \beta$ fluxes adopted from Peterson et al. (2000), are given in Table 1. Column (1) lists the file name, and Col. (2) the corresponding Julian Date of observation. The continuum and $\mathrm{H} \beta$ fluxes, both measured by Peterson et al. (2000), are listed in Cols. (4) and (5), respectively. Column (6) is the flux of the subtracted optical Fe II complex between rest wavelength $4434 \AA$ and $4684 \AA$, along with the determined upper and lower limits. The modelled total flux of $\mathrm{H} \beta$ is given in Col. (7), and the total flux of He II $\lambda 4686$ in Col. (8). All the errors given in Cols. (7) and (8) are caused by profile modelling.

The correlation between the modelled flux of $\mathrm{H} \beta$ and the flux provided by Peterson et al. (2000) is illustrated in Fig. 4. The modelled $\mathrm{H} \beta$ flux containing all three components is represented by solid square symbols (Correlation I, for short), and the modelled $\mathrm{H} \beta$ flux in which the very broad component are excluded, by open triangles (Correlation II, for short). Statistical analysis yields a Spearman rank-order correlation coefficient $r_{\mathrm{s}}=0.896\left(P<10^{-4}\right.$, where $P$ is the probability that there is null relation between two variables) for Correlation I and $r_{\mathrm{s}}=0.708\left(P<10^{-4}\right)$ for Correlation II. Because of the lower inferred correlation coefficient for Correlation II with

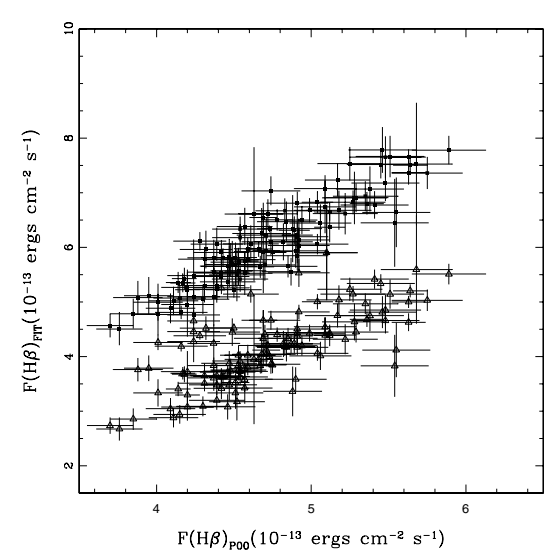

Fig. 4. The correlation between two independent $\mathrm{H} \beta$ measurements (see text for details). The horizontal axis is the value obtained by Peterson et al. (2000), and the vertical one the value provided by profile modelling. The total flux including all three components is denoted by solid squares $\left(r_{\mathrm{s}}=0.896, P<10^{-4}\right)$, while the flux, including a narrow peak and a classical broad component, is denoted by open triangles $\left(r_{\mathrm{s}}=0.708, P<10^{-4}\right)$.

respect to that for Correlation I, we clarify that the very broad component should not be ignored in accounting for the total flux of $\mathrm{H} \beta$. Systematically, the modelled flux is slightly larger in this way than the flux provided by Peterson et al. (2000). This systematical enhancement can be explained easily by emission at the high velocity wing of the very broad base. In the study by Peterson et al. (2000), the $\mathrm{H} \beta$ high velocity wing is truncated by integration ranging from $4820 \AA$ to $4910 \AA$ in the observed frame. Comparing the flux modelled independently in this paper to the one obtained in Peterson et al. (2000), we find a highly significant, nearly linear correlation between them.

\subsection{Light curves}

The derived light curves of $\mathrm{H} \beta$, He II $\lambda 4686$, and Fe II are displayed in the bottom three panels in Fig. 5. Moreover, the light curves of $\mathrm{H} \beta$ and continuum both derived by Peterson et al. (2000) are shown in the top two panels. The two independent $\mathrm{H} \beta$ light curves are quite similar. The second panel, from bottom to top, shows the light curve of the He II line. Here, it should be emphasized that the flux of He II is obtained from the Fe II contamination-removed spectrum. Because of the significant blending between He II and the Fe II complex, the Fe II emission is a complicating factor when measuring the strength of He II line. In Fig. 5, the error bars overlaid on the He II light curve include only those uncertainties caused by profile modelling and do not reflect the errors caused by the Fe II subtraction. The Fe II variations are shown in the bottom panel of Fig. 5. The length of each overlaid solid line corresponds to the range between upper and lower limits, which are determined by the iterative experiments of the Fe II subtraction. The pattern of the Fe II variations closely follows the continuum (and $\mathrm{H} \beta$ ) light curve. Despite all our efforts, any significant lag of the Fe II complex with respect to the continuum could not be determined because of the large uncertainties of the Fe II flux. 


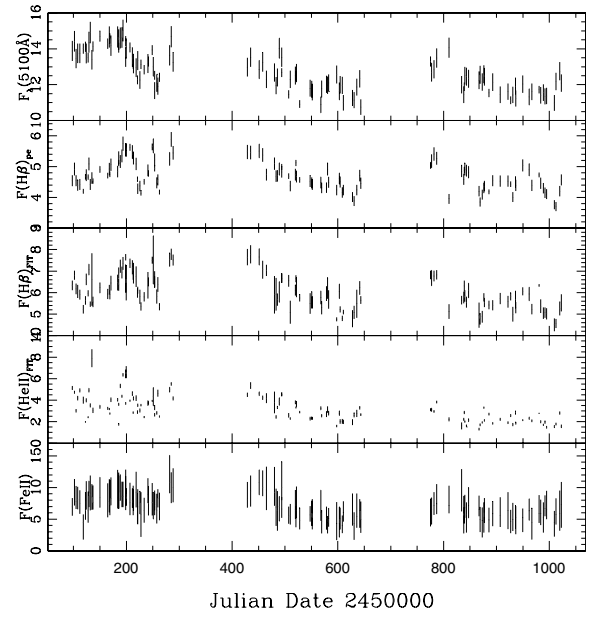

Fig. 5. Light curves of continuum and emission lines for NGC 4051 with 120 points taken between Jan. 15, 1996 and Jul. 28, 1998. The first panel shows the light curve of continuum in units of $10^{-15} \mathrm{erg} \mathrm{s}^{-1} \mathrm{~cm}^{-2} \AA$. The $\mathrm{H} \beta$ light curve adopted from Peterson et al. (2000) is displayed in the second panel in units of $10^{-13} \mathrm{erg} \mathrm{s}^{-1} \mathrm{~cm}^{-2}$. The third, fourth, and fifth panels illustrate, respectively, the emission line light curves of $\mathrm{H} \beta, \mathrm{He}$ II $\lambda 4686$, and Fe II in units of $10^{-13} \mathrm{erg} \mathrm{s}^{-1} \mathrm{~cm}^{-2}$.

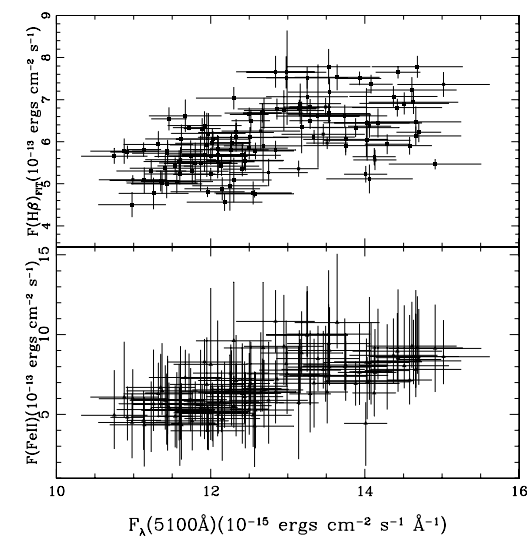

Fig. 6. Lower panel: line intensity of the optical Fe II blends plotted against continuum at rest wavelength $5100 \AA\left(r_{\mathrm{s}}=0.701, P<10^{-4}\right)$. In addition, intensity of $\mathrm{H} \beta$ as function of continuum flux is shown in the upper panel $\left(r_{\mathrm{s}}=0.564, P<10^{-4}\right)$.

In the lower panel of Fig. 6, the flux of the Fe II complex is plotted as a function of continuum flux as an additional test of line variability. The diagram shows a positive correlation between Fe II intensity and continuum flux $\left(r_{\mathrm{s}}=0.701\right.$, $\left.P<10^{-4}\right)$. The relationship of the $\mathrm{H} \beta$ line with respect to continuum is displayed in the upper panel $\left(r_{\mathrm{s}}=0.564, P<10^{-4}\right)$.

\subsection{Equivalent width and RFe}

The Baldwin Effect (BE) defined as an anti-correlation between the equivalent width $(E W)$ of C IV $\lambda 1549$ and continuum luminosity at $\lambda 1450$ was first reported by Baldwin (1977). Subsequent observational studies indicated that the BE can be detected in almost all measurable high ionization emission lines (Espey et al. 1993; Lanzetta et al. 1993; Zheng \& Malkan 1993) except N Vג1240 (Hamann \& Ferland 1999).

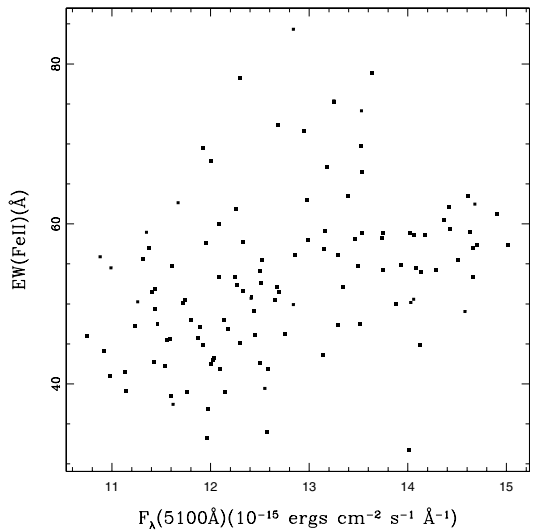

Fig. 7. A plot of equivalent width for the optical Fe II complex against the flux of continuum $\left(r_{\mathrm{s}}=0.384, P<10^{-4}\right)$.

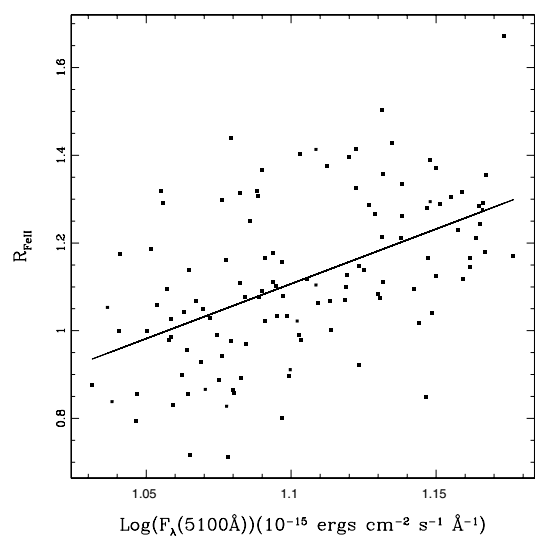

Fig. 8. $R_{\mathrm{Fe}}$ plotted against the logarithm of continuum flux $\left(r_{\mathrm{s}}=\right.$ $\left.0.521, P<10^{-4}\right)$. The best fitted relation $R_{\mathrm{Fe}} \propto(5.0 \pm 0.8) \log (L / M)$ is shown by the overlaid solid curve.

Gilbert \& Peterson (2003) recently found a convincing intrinsic $\mathrm{BE}$ in the broad $\mathrm{H} \beta$ line of the active galaxy NGC 5548 by analyzing spectra from International AGN Watch collaboration (see also in Goad et al. 2004).

In Fig. 7, the $E W$ of the Fe II complex is plotted against continuum flux. There is a moderate correlation between these data $\left(r_{\mathrm{s}}=0.384, P<10^{-4}\right.$ ), which confirms previous claims that no convincing BE has been detected in the optical Fe II blends (Yee \& Oke 1981; Elston et al. 1994). The relationship is influenced slightly by a few points with excessive Fe II contributions. These points clearly deviate from the other points. Detailed inspection of the light curves indicates that these specific points are mainly deduced from spectra observed from $\mathrm{JD}=2450193.6$ to 2450465.0 . Subsequently, the corresponding original spectra, Fe II subtraction, and profile modelling are carefully inspected. We find that the deduced excessive Fe II emission is perhaps caused by the lower $S / N$ ratio at blueward of the spectra. The bad quality of the spectra makes measurement of the Fe II blends difficult and ultimately leads to an over-removal of the Fe II complex because of blending between Fe II and other faint emission lines, such as He I $\lambda 4471$.

The intrinsic variations of $R_{\mathrm{Fe}}$ as a function of continuum flux are plotted in Fig. $8 . R_{\mathrm{Fe}}$ is one of the most important quantities describing the E1 parameter space, and it 


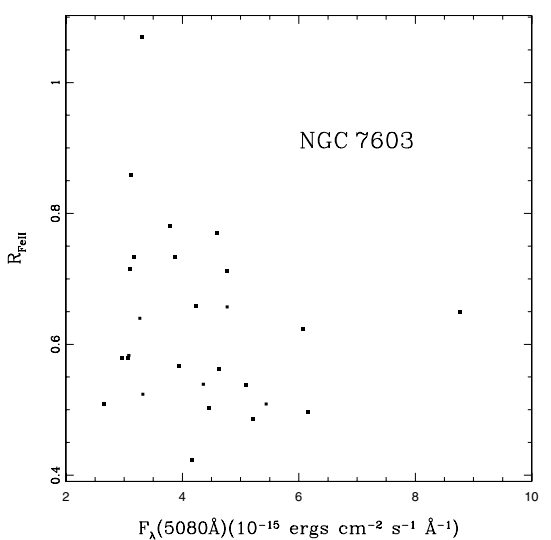

Fig. 9. A plot of $R_{\mathrm{Fe}}(4570 \AA)$ against the continuum flux in NGC 7603 . The fluxes are measured by Kollatschny et al. (2000). $R_{\mathrm{Fe}}$ is calculated by flux ratio between Fe II and $\mathrm{H} \beta$.

is defined as the flux ratio of optical Fe II complex to $\mathrm{H} \beta$. Although the flux of $\mathrm{H} \beta$ includes the contributions of narrow, broad, and very broad components, it should be noted that the flux of the narrow component of $\mathrm{H} \beta$ is, of course, expected to be constant. This plot shows a positive correlation between $R_{\mathrm{Fe}}$ and continuum flux logarithm. The correlation coefficient derived by Spearman analysis is $r_{\mathrm{s}}=0.521\left(P<10^{-4}\right)$. An unweighted linear fit to this relation gives $R_{\mathrm{Fe}} \propto(2.5 \pm$ $0.4) \log \left(\left[F_{\lambda}(5100 \AA) / 10^{-13} \mathrm{erg} \mathrm{s}^{-1} \mathrm{~cm}^{-2}\right]\right)$ and is over-plotted in Fig. 8 as a solid line. By analyzing the reverberation mapping (e.g. Blandford \& Mckee 1982; Peterson et al. 1998b) results of spectrophotometrical monitoring of a well-defined sample of 17 Palomar Green quasars and 17 Seyfert galaxies, Kaspi et al. (2000) found that the relation between the Eddington ratio and the continuum luminosity at $\lambda 5100$ can be expressed as $L / L_{\mathrm{E}} \propto\left[\lambda L_{\lambda}(5100 \AA) / 10^{44} \mathrm{erg} \mathrm{s}^{-1}\right]^{0.5}$. Consequently, by combining the above relationships, we find that $R_{\mathrm{Fe}}$ can be inferred to scale with the Eddington ratio as $R_{\mathrm{Fe}} \propto(5.0 \pm 0.8) \log (L / M)$. Our relationship does not agree with that found by Marziani et al. (2001, and references therein). To predict a grid of theoretical values in E1 parameter space, these authors obtained a global semi-theoretical relation between $R_{\mathrm{Fe}}$ and the Eddington ratio: $R_{\mathrm{Fe}} \propto 0.55 \log (L / M)$. The discrepancy between the two relations is highly significant.

\section{Discussion: Comparison of variability in Fe Il complex: NGC 4051 and other AGNs}

In this paper, we detect a positive correlation between $R_{\mathrm{Fe}}$ and the continuum flux in NGC 4051. However, it should be emphasized that the real situation is hard to handle when taking other results about variations of Fe II emission into account. For instance, by calculating the flux ratio of $\mathrm{Fe}$ II to $\mathrm{H} \beta$, we find that in NGC 7603, $R_{\mathrm{Fe}}$ apparently decreases with increasing continuum (Kollatschny et al. 2000). This relation is displayed in Fig. 9. The point observed at JD $=24044168$ is omitted because it clearly deviates from the other points. The difference in variability behavior of $R_{\mathrm{Fe}}$ implies that the Seyfert galaxies NGC 4051 and NGC 7603 differ in those physical conditions that govern the variability behavior of Fe II emission.
Table 2. Variations of the Fe II complex in six selected Seyfert galaxies.

\begin{tabular}{cccc}
\hline \hline Source name & $\begin{array}{c}F W H M^{a} \\
\left(\mathrm{~km} \mathrm{~s}^{-1}\right) \\
(2)\end{array}$ & $R_{\mathrm{Fe}}$ vs. $F_{\lambda}^{b}$ & Reference \\
\hline NGC $7603 .$. & 6560 & Negative & $(4)$ \\
Mark $110 \ldots$ & 1515 & Negative & 2 \\
NGC $4051 \ldots$ & 1100 & Positive & $\ldots$. \\
Mark $359 \ldots$ & 900 & Positive & 3,4 \\
Mark1044.. & 1010 & Positive & 3,4 \\
Akn $564 \ldots$ & 865 & Positive & 3,4 \\
\hline
\end{tabular}

${ }^{a}$ Each of the FWHM measured from the mean profile of the $\mathrm{H} \beta$ line.

${ }^{b}$ The relation between $R_{\mathrm{Fe}}$ and continuum. Positive: the relation is positive; Negative: the relation is negative.

Notes 1: Kollatschny et al. (2000); 2: Kollatschny et al. (2001); 3: Giannuzzo \& Stirpe (1996); 4: Véron-Cetty et al. (2001).

In order to statistically investigate the behavior of Fe II variations, we collected some results about Fe II variations from earlier publications. The comparison is summarized in Table 2. Column (1) lists the object name, and Col. (2) the averaged $F W H M$ of $\mathrm{H} \beta$. Column (3) summarizes the relation between $R_{\mathrm{Fe}}$ and continuum flux. In fact, whether a positive or a negative correlation can be obtained is determined by the fact that the variations of the Fe II blends are stronger or weaker in comparison to the $\mathrm{H} \beta$ line. In Mark 359, Mark 1044, and Akn 564, the percentage variations in line fluxes of $\mathrm{H} \beta$ and Fe II $\lambda 4550$ were given by Giannuzzo \& Stirpe (1996). The variability behavior of $R_{\mathrm{Fe}}$ could be easily obtained in terms of the ratio between the percentage variation of Fe II and that of $\mathrm{H} \beta$. If the ratio is larger than unity, then $R_{\mathrm{Fe}}$ is expected to increase with continuum. Instead, $R_{\mathrm{Fe}}$ decreases with continuum when the ratio is less than 1 . In NLS1 galaxy Mark $110, R_{\mathrm{Fe}}$ is expected to decrease with continuum flux both because the $\mathrm{H} \beta$ line varied by a factor of about 2 and because the Fe II lines remained constant over the 10 years.

By comparing the variability behaviors of different objects, we find that all objects with positive correlations have narrow $\mathrm{H} \beta$ profiles and can be classified as NLS1s. In contrast, the remaining two sources with negative correlations have relatively broad $\mathrm{H} \beta$ profiles (i.e. $F W H M>1500 \mathrm{~km} \mathrm{~s}^{-1}$ ). The dichotomy in variability behavior of $R_{\mathrm{Fe}}$ suggests that the variability amplitude of the Fe II complex in Seyfert galaxies might be correlated with the width of the $\mathrm{H} \beta$ line. Because the standard photoionization models cannot interpret the strong Fe II emission, we attempt to interpret the dichotomy in variability behavior of $R_{\mathrm{Fe}}$ in the framework of collisional models, in which the bulk excitation of the optical Fe II lines is due to collisional excitation in a high density optically thick cloud illuminated and heated mainly by X-rays photons (Wills et al. 1985; Kwan et al. 1995; Sigut \& Pradhan 2003; Verner et al. 1999; Collin-Souffrin et al. 1986, 1988). The Fe II emission region is typical of $N_{\mathrm{e}} \sim 10^{10-12} \mathrm{~cm}^{-3}, N_{\mathrm{H}}>10^{24} \mathrm{~cm}^{-2}$, and $T_{\mathrm{e}} \sim 8000 \mathrm{~K}$.

We discuss the observed Fe II variability in terms of the extensively used line responsivity $\partial j_{1}(t) / \partial F_{\mathrm{c}}$, where $j_{1}$ is the 
emissivity of a given line and $F_{\mathrm{c}}$ the incident ionizing continuum flux. The time dependent responsivity means that the gas requires some time to equilibrate to a new continuum level. We clarify that this delay, in minutes, is so short that it can be neglected on the basis of the following discussions. Relaxation to thermal balance takes place on the timescale $t_{\text {cool }} \sim 5 \times 10^{11} n_{\mathrm{e}}^{-1} \mathrm{~s}$ (Krolik 1999), where the fact that the cooling function is usually $\sim 10^{-23} \mathrm{erg} \mathrm{cm}^{3} \mathrm{~s}^{-1}$ when the temperature is around $10^{4} \mathrm{~K}$ is used. This formula provides a cooling timescale of about $0.1-1000 \mathrm{~s}$ when typical values of density in BLR are taken (e.g. in NGC $4051 N_{\mathrm{e}} \sim 10^{10} \mathrm{~cm}^{-3}$, Hyung et al. 2000; $8.7<\log N_{\mathrm{e}}<9.1$, Komossa \& Mathur 2001). These calculations indicate that, relative to the timescales on which the intrinsic continuum changes (weeks to years), the cooling timescale can be entirely ignored.

Now we focus attention on the line responsivity $\partial j_{1} / \partial F_{\mathrm{c}}$, and consider the two possible explanations:

1. Line responsivity as a function of electron density. Recent numerical calculations have indicated that the responsivity of the Fe II line flux in higher density case is much larger than in the case with lower density (see Fig. 6 in Sigut et al. 2004). The Fe II flux is enhanced by about one order in the model with $\log n_{\mathrm{H}}=9.5$, but by nearly two orders in the model with $\log n_{\mathrm{H}}=11.5$, when the ionization parameter increases from $10^{-3}$ to $10^{-1.5}$. Since there is a significant correlation between $R_{\mathrm{Fe}}$ and electron density (Aoki \& Yoshida 1999; Wills et al. 1999; Marziani et al. 2001, and references therein), adopting the generally accepted E1 correlation $R_{\mathrm{Fe}} \propto F W H M^{-1}$ yields $F W H M(\mathrm{H} \beta) \propto n_{\mathrm{e}}^{-1}$. For example, in IZW1 the line ratio Si III] $\lambda 1892 / C$ III] $\lambda 1909 \approx 3.5$ (Laor et al. 1997) is much larger than the typical value for quasars $(\approx 0.3$, Laor et al. 1995). The ratio $\mathrm{Si} \mathrm{III]/C} \mathrm{III]} \mathrm{is} \mathrm{a} \mathrm{useful} \mathrm{density} \mathrm{diagnostic}$ in BLR (Ferland et al. 2000). In general, the responsivity of the Fe II lines in NLS1 is consequently expected to be larger than in Broad-Line Seyfert 1 galaxy (BLSy1).

2. Line responsivity as a function of incident continuum shape. It is clear that the reprocessed spectrum also depends on the shape of incident continuum. Continuum energies that should most affect Fe II strength are $h v>800 \mathrm{eV}$ (Krolik \& Kallman 1988). Recently, the positive correlations between ROSAT HR1 and Count Rates were identified for six out of eight NLS1s, but the anti-correlations were identified for seven out of 14 BLSy1s (Cheng et al. 2002). There were no detectable correlations in the other two NLS1s and 7 BLSy1s. This means that, in general, the fraction of ionizing photons at high energy level increases with incident continuum in NLS1. In contrast, a decreased fraction can usually be found in BLSy1. If so, the magnitude of changes in heat caused by the high energy photons are stronger in NLS1 and weaker in BLSy1, so that the observed variations of the optical Fe II blends logically become strong in NLS1 and weak in BLSy1.

In summary, the dichotomy in the variability behavior of $R_{\mathrm{Fe}}$ could be caused by one of two different physical conditions, i.e. either by electron density in a single cloud or by variability behavior of incident high energy photons. At present, however, evidence is not conclusive enough to determine which of them is more important. Although we discuss them separately, it is also possible for both mechanisms to act together in AGN. It might be worthwhile to extend variability campaigns to larger Seyfert samples with different broad line widths in order to investigate the validity of the trend mentioned above. It is also likely that complicated photoionization model calculations are necessary to distinguish between the proposed two interpretations. However, this model study is beyond the scope of this paper.

\section{Conclusions}

We perform new analysis of the archival spectra of NGC 4051 extracted from the AGN Watch project in order to investigate the variability of optical Fe II emission. The template of BG92 is used to remove and to measure the Fe II complex. The other emission lines are profiled by multi-component profile modelling. This analysis allows us to make the following conclusions:

1. In NGC 4051, we find that the optical Fe II complex was variable during the three years period. The Fe II variations closely follow the continuum variations, and the intensity of Fe II evidently increases with the continuum flux.

2. A positive correlation between the $E W$ of Fe II and the continuum flux is identified in NGC 4051. This result agrees with the previous claims that no convincing BE of Fe II has been detected until now.

3. By comparing the variations of $\mathrm{H} \beta$ and Fe II, a positive correlation between $R_{\mathrm{Fe}}$ and continuum flux is obtained in NGC 4051. The unweighted fit gives the relation $R_{\mathrm{Fe}} \propto$ $(5.0 \pm 0.8) \log (L / M)$. This relation is significantly different from the relation $R_{\mathrm{Fe}} \propto 0.55 \log (L / M)$, predicted by the semi-theoretical formula (Marziani et al. 2000).

4. We find an inverse correlation between $R_{\mathrm{Fe}}$ and continuum in Seyfert galaxy NGC 7603 (Kollatschny et al. 2000). The difference in the variability behavior of $R_{\mathrm{Fe}}$ implies that the Seyfert galaxies NGC 4051 and NGC 7603 differ in physical conditions governing the variability of the optical Fe II blends. Furthermore, in six selected Seyfert galaxies, the positive correlations are identified in 4 out of 5 NLS1s and for the negative correlations, in the remaining two objects whose $\mathrm{H} \beta$ profiles are relatively broad. The different electron density of BLR clouds and variability behavior of high energy photons are put forward to interpret the dichotomy in variability behavior of $R_{\mathrm{Fe}}$.

Acknowledgements. We are grateful to the anonymous referee for many useful suggestions. The authors acknowledge many valuable discussions with Dr. Xu, D. W., Hao, C. N., S. Komossa, and Mao, Y. F. This work has made use of the archival spectroscopic data of the AGN Watch campaign. We thank Prof. Bradley M. Peterson for providing a grant for using the spectra of NGC 4051. Our thanks also go to Dr. Todd A. Boroson and Richard F. Green for providing the Fe II template. This work was financially supported by the Ministry of Science and Technology of China, under grant NKBRSF G19990754, and by the NSF of China, No. 10473013. 


\section{References}

Aoki, K., \& Yoshida, M. 1999, ASP Conf. Ser., 162, 385

Baldwin, J. A. 1977, ApJ, 214, 679

Blandford, R. D., \& Mckee, C. F. 1982, ApJ, 255, 419

Boroson, T. A. 2002, ApJ, 565, 78

Boroson, T. A., \& Green, R. F. 1992, ApJS, 80, 109

Christopoulou, P. E., Holloway, A. J., Steffen, W., et al. 1997, MNRAS, 284, 385

Collin-Souffrin, S., Joly, M., Pequignot, D., \& Dumont, S. 1986, A\&A, 166, 27

Collin-Souffrin, S., Hameury, J.-M., \& Joly, M. 1988, A\&A, 205, 19

Cheng, L. P., Wei, J. Y., \& Zhao, Y. H. 2002, ChJAA, 2, 207

Davidson, K., \& Netzer, H. 1979, RvMP, 51, 715

Dietrich, M., Kollatschny, W., Peterson, B. M., et al. 1993, ApJ, 408, 416

Dultzin-Hacyan, D. 1987, Rev. Mex. Astron. Astrofis., 14, 94

Elston, R., Thompson, K. L., \& Hill, G. J. 1994, Nature, 367, 250

Espey, B. R., Lanzetta, K. M., \& Turnshek, D. A. 1993, A\&AS, 18310606

Evans, I. N. 1988, ApJS, 67, 373

Ferland, G. J. 2000, in Astrophysical Plasmas: Codes, Models, and Observations, ed. J. Arthur, N. Brickhouse, \& J. Franco, Rev. Mex. Astron. Astrofis. Ser. Conf., 9 (México, D. F.,: Inst. Astron., Univ. Nac. Autónoma México), 153

Giannuzzo, E. M., \& Stirpe, G. M. 1996, 314, 419

Gilbert, K. M., \& Peterson, B. M. 2003, ApJ, 587, 123

Goad, M. R., Korista, K. T., \& Knigge, C. 2004, MNRAS, 352, 277

Graham, M. J., Clowes, R. G., \& Campusano, L. E. 1996, MNRAS, 279,1349

Hamann, F., \& Ferland, G., ARA\&A, 37, 487

Heckman, T. M., Miley, G. K., van Breugel, W. J. M., \& Butcher, H. R. 1981, ApJ, 247, 403

Hyung, S., Kim, H., Lee, W. B., et al. 2000, JKAS, 33, 81

Joly, M. 1987, A\&A, 184, 33

Joly, M. 1991, A\&A, 242, 49

Jarvis, M. J., \& McLure, R. J. 2002, MNRAS, 336, L38

Kaspi, S., Smith, P. S., Netzer, H., et al. 2000, ApJ, 533, 631

Kollatschny, W., Bischoff, K., \& Dietrich, M. 2000, A\&A, 361, 901

Kollatschny, W., Bischoff, K., Robinson, E. L., Welsh, W. F., \& Hill, G. J. 2001, A\&A, 379, 125

Kollatschny, W., \& Fricke, K. J. 1985, A\&A, 146, L11

Kollatschny, W., Schleicher, H., Fricke, K. J., \& Yorke, H. W. 1981, A\&A, 104, 198

Komossa, S., \& Mathur, S. 2001, A\&A, 374, 914

Korista, K., Baldwin, J., Ferland, G., \& Verner, D. 1997, ApJS, 108, 401

Korista, K., \& Goad, M. 2004, ApJ, 606, 749

Kriss, G. 1994, ASPC, 61, 437

Krolik, J. H. 1999, Active Galactic Nuclei (Princeton, NJ: Princeton Univ. Press)

Krolik, J. H., \& Kallman, T. R. 1988, ApJ, 324, 714

Kwan, J., \& Krolik, J. H. 1981, ApJ, 250, 478
Kwan, J. 1984, ApJ, 283, 70

Kwan, J., Cheng, F.-Z., Fang, L.-Z., Zheng, W., \& Ge, J. 1995, ApJ, 440,628

Lanzetta, K. M., Turnshek, D. A., \& Sandoval, J. 1993, ApJS, 84, 109

Lawrence, A., Saunders, W., Rowan-Robinson, M., et al. 1988, MNRAS, 235, 261

Laor, A., Jannuzi, B. T., Green, R. F., \& Boroson, T. A. 1997, ApJ, 489, 656

Laor, A., Bahcall, J. N., Jannuzi, B. T., Schneider, D. P., \& Green, R. F. 1995, ApJS, 99, 1

Lipari, S. 1994, ApJ, 436, 102

Marziani, P., Sulentic, J. W., Zwitter, T., Dultzin-Hacyan, D., \& Calvani, M. 2001, ApJ, 558, 553

Marziani, P., Sulentic, J. W., Zamanov, R., \& Calvani, M. 2003b, Mem. S. A. It., 74, 490

Marziani, P., Zamanov, R. K., Sulentic, J. W., \& Calvani, M. 2003a, MNRAS, 345, 1133

Moran, E. C., Halpern, J. P., \& Helfand, J. 1996, ApJS, 106, 341

Netzer, H., \& Wills, B. J. 1983, ApJ, 275, 445

Oke, J. B., \& Lauer, T. R. 1979, ApJ, 230, 360

Osterbrock, D. E., \& Pogge, R. W. 1985, ApJ, 297, 166

Peterson, B. M., McHardy, I. M., Wilkes, B. J., et al. 2000, ApJ, 542, 161

Peterson, B. M., Wanders, I., Bertram, R., et al. 1998, ApJ, 501, 82

Phillips, M. M. 1978, ApJ, 226, 736

Sigut, T. A. A., \& Pradhan, A. K. 1998, ApJL, 499, 139

Sigut, T. A. A., \& Pradhan, A. K. 2003, ApJS, 145, 15

Sigut, T. A. A., Pradhan, A. K., \& Nahar, S. N. 2004, ApJ, 611, 81

Stasinska, G. 1984, A\&AS, 55, 15

Storey, P. J., \& Zeippen, C. J. 2000, MNRAS, 312, 813

Sulentic, J. W., Marziani, P., \& Dultzin-Hacyan, D. 2000a, ARA\&A, 38,521

Sulentic, J. W., Marziani, P., Zwitter, T., Dultzin-Hacyan, D., \& Calvani, M. 2000c, ApJ, 545, L15

Sulentic, J. W., Zwitter, T., Marziani, P., \& Dultzin-Hacyan, D. 2000b, ApJ, 536, L5

Verner, E. M., Verner, D. A., Korista, K. T., et al. 1999, ApJS, 120, 101

Véron-Cetty, M.-P., Véron, P., \& Goncalves, A. C. 2001, A\&A, 372, 730

Véron-Cetty, M.-P., Joly, M., \& Véron, P. 2004, A\&A, 417, 515

Wills, B. J., Netzer, H., \& Wills, D. 1985, ApJ, 288, 94

Wills, B. J., Laor, A., Brotherton, M. S., et al. 1999, ApJ, 515, L53

Xia, X.-Y., Mao, S., Wu, H., et al. 1999, A\&A, 341, L13

Xu, D. W., Komossa, S., Wei, J. Y., Qian, Y., \& Zheng, X. Z. 2003, ApJ, 590, 73

Yee, H. K. C., \& Oke, J. B. 1981, ApJ, 248, 472

Zamanov, R., \& Marziani, P. 2002, ApJ, 571, L77

Zamanov, R., Marziani, P., Sulentic, J. W., et al. 2002, ApJ, 576, L9

Zheng, W., \& Malkan, M. A. 1993, ApJ, 415, 517

Zhou, H. Y., Wang, T. G., Zhou, Y. Y., Li, C., \& Dong, X. B. 2002, ApJ, 581, 96 